\title{
p53-responsive TLR8 SNP enhances human innate immune response to respiratory syncytial virus
}

\author{
Daniel Menendez,, ${ }^{1,2}$ Joyce Snipe, ${ }^{1}$ Jacqui Marzec, ${ }^{2}$ Cynthia L. Innes, ${ }^{3}$ Fernando P. Polack, ${ }^{4}$ Mauricio T. Caballero, ${ }^{4}$ \\ Shepherd H. Schurman, ${ }^{3}$ Steven R. Kleeberger, ${ }^{2}$ and Michael A. Resnick ${ }^{1}$ \\ ${ }^{1}$ Cenome Integrity \& Structural Biology Laboratory, ${ }^{2}$ Immunity, Inflammation, and Disease Laboratory and ${ }^{3}$ Clinical Research Branch, National Institute of Environmental Health Sciences, Research Triangle \\ Park, North Carolina, USA. ${ }^{4}$ Fundación INFANT, Buenos Aires, Argentina.
}

\begin{abstract}
The Toll-like receptor 8 (TLR8) has an important role in innate immune responses to RNA viral infections, including respiratory syncytial virus (RSV). We previously reported that TLR8 expression was increased directly by the tumor suppressor and transcription factor p53 via a single nucleotide polymorphism (SNP) (rs3761624) in the TLR8 promoter, thereby placing TLR8 in the p53/immune axis. Because this SNP is in linkage disequilibrium with other SNPs associated with several infectious diseases, we addressed the combined influence of p53 and the SNP on downstream inflammatory signaling in response to a TLR8 cognate ssRNA ligand. Using human primary lymphocytes, p53 induction by chemotherapeutic agents such as ionizing radiation caused SNP-dependent synergistic increases in IL-6 following incubation with an ssRNA ligand, as well as TLR8 RNA and protein expression along with p53 binding at the TLR-p53 SNP site. Because TLR8 is X-linked, the increases were generally reduced in heterozygous females. We found a corresponding association of the p53-responsive allele with RSV disease severity in infants hospitalized with RSV infection. We conclude that p53 can strongly influence TLR8mediated immune responses and that knowledge of the p53-responsive SNP can inform diagnosis and prognosis of RSV disease and other diseases that might have a TLR8 component, including cancer.
\end{abstract}

\section{Introduction}

The human innate immune system provides early and effective responses against many invading pathogens and potential environmental threats. A critical component of innate immunity is pattern recognition of a wide range of molecules, an important factor in determining the outcome of infection. Detection by sensors of the innate immune system or intrinsic restriction of viruses can lead to expression of key cytokines and chemokines that direct and amplify host immune responses $(1,2)$.

Beyond its classical function as guardian of the genome via the modulation of genes involved in cell-cycle arrest, apoptosis, DNA repair and senescence, the p53 stress-induced transcription factor has recently been found to participate in other processes, including the immune response. Besides transcriptional upregulation of TP53 by interferons in response to viral infections (3), the expression of many immune genes, including those of the innate immune toll-like receptor (TLR) (4) and apolipoprotein B mRNA-editing enzyme, catalytic polypeptide-like type 3 (APOBEC3) (5) gene families are subject to direct p53 regulation. The presence of p53 response elements (p53REs) associated with the transcriptional regulation of TLR and APOBEC3 genes as well as other interferon-stimulated genes (ISGs) such as

Conflict of interest: DM, SRK, FPP, and MAR are inventors of a patent pending for use of a TLR8-associated SNP in prognosing and treating individuals who have a microbial infection (patent application no. 62/881,656).

Copyright: () 2019, American Society for Clinical Investigation.

Submitted: March 8, 2019; Accepted: August 8, 2019; Published: October 7, 2019.

Reference information: J Clin Invest. 2019;129(11):4875-4884.

https://doi.org/10.1172/JCl128626.
IRF9, IRF5, and ISG15, identifies p53 as a central mediator and amplifier of global innate immune responses (6). In addition, we recently determined by meta-analysis of p53 binding and associated gene expression that $\mathrm{p} 53$ transcriptionally regulates several immune/inflammatory genes (7). Together, these studies support an extensive $\mathrm{p} 53$ /immune axis in humans as guardian of the immune integrity (6).

TLRs are in a family of transmembrane proteins involved in the first line of defense against infections and are expressed on a variety of immune sensing cells. These pattern recognition receptors recognize a variety of pathogen-associated molecular patterns (PAMPs) (8). In humans, there are 10 TLRs that identify different PAMPs (9). TLR8, which is located in endosomes, recognizes guanosine- or uridine-rich single-stranded RNA (ssRNA) from viruses such as human immunodeficiency virus, vesicular stomatitis virus, and many respiratory viruses (10). TLR8 also influences bacterial infection (11) and autoimmunity (12).

We found that activated p53 can target TLR8 expression in a single nucleotide polymorphism-dependent (SNP-dependent) manner. The SNP rs3761624 situated in the p53RE of the $T L R 8$ promoter region $(4,13)$ increases TLR 8 mRNA expression following acute and chronic DNA damage stress in human primary $\mathrm{T}$ lymphocytes and alveolar macrophages in a p53RE SNPdependent manner, where the minor $G$ allele of the A/G SNP pair is p53 responsive. This allele creates a perfect CWWG core in the second decamer of the p53RE within the TLR8 promoter (Figure 1A). The CWWG motif is highly conserved in p53 binding sites (7). A change in the conserved $\mathrm{C}$ or $\mathrm{G}$ in the RE dramatically affects transactivation $(13,14)$. The presence of the A allele in the rs3761624 variant disrupts the CWWG core, reducing p53 binding. 
A

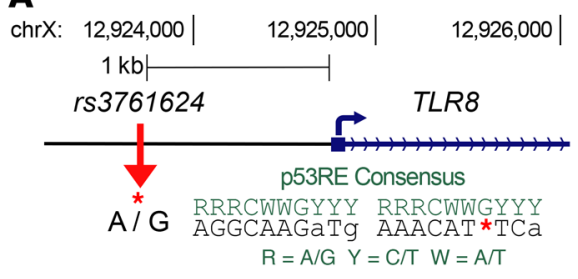

B
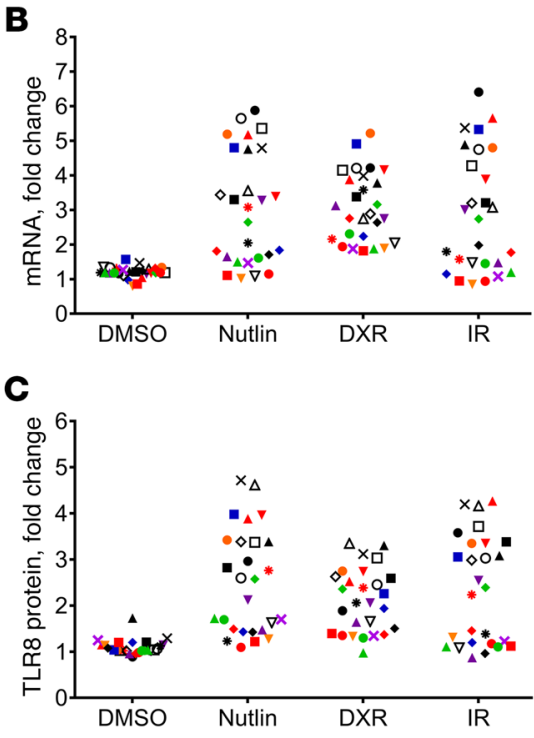

D

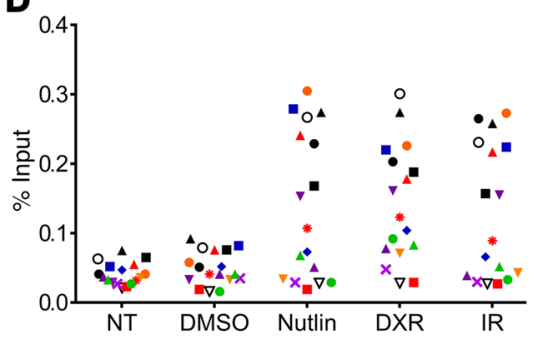

\section{E}
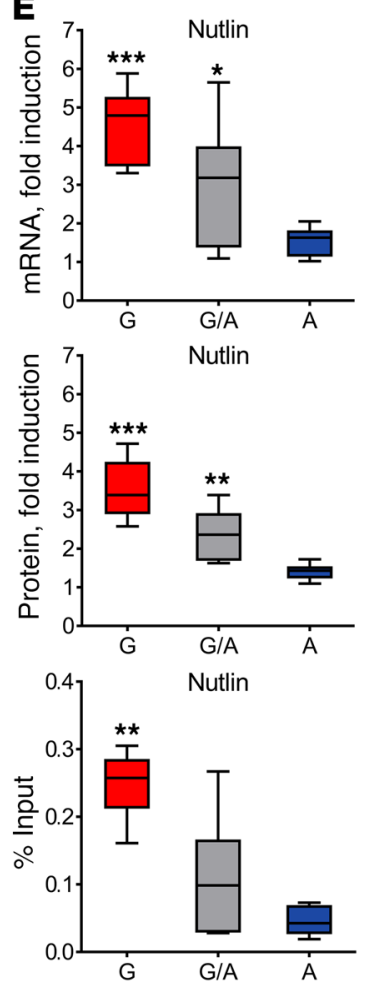

$\mathbf{F}$
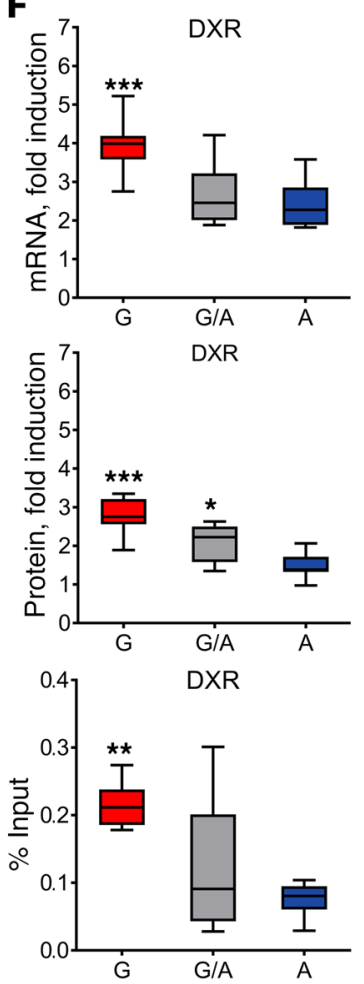

G
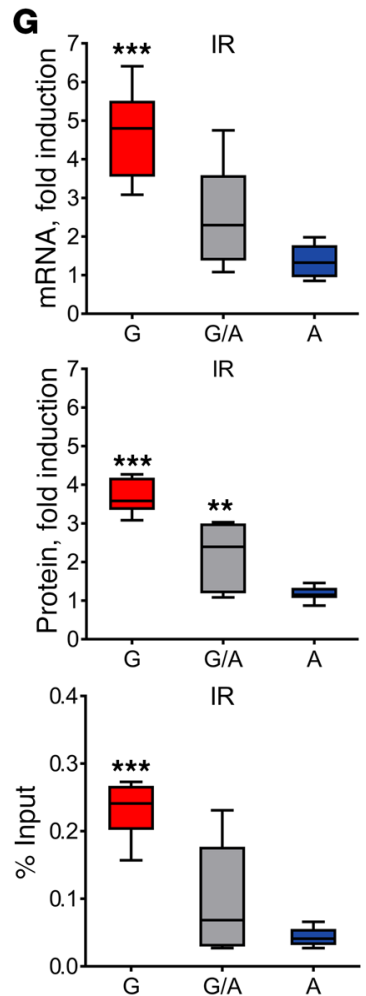

Figure 1. TLR8 gene responsiveness to $p 53$ activation by chemotherapeutic drugs is SNP rs3761624 dependent. (A) Graphical location of TLR8 p53-SNP rs3761624 (A/G*) relative to the TSS of the gene and to the p53 Response Element (p53RE). Blinded TLR8 gene (B) and protein (C) expression and (D) p53 occupancy profiles in human lymphocytes after 24 hours of treatment with p53 activators nutlin $(10 \mu M)$, DXR $(1 \mu M)$, and IR (4 Gy). Each dot represents a different donor. A total of 27 donors were evaluated for gene and protein expression, and 17 for occupancy. Presented in (E) nutlin, (F) DXR, and (G) IR are the decoded 24-hour results for TLR8 mRNA $(n=25)$ and protein $(n=25)$ expression profiles and p53 occupancy $(n=16)$ grouped by rs3761624 A/ $G$ genotypes. The horizontal bars represent the mean values. ${ }^{*} P<0.05$; ${ }^{* *} P<0.01 ;{ }^{* *} P<0.0001$ (2-tailed unpaired Student's $t$ test).

We hypothesized that activation of p53 can differentially enhance the TLR8 innate immune-mediated responses in human lymphocytes depending on the TLR8 p53RE SNP genotype. Here, we investigate the influence of p53 activation on the functional effects of the TLR 8 rs 3761624 variant and its impact on TLR8 signaling in peripheral blood $\mathrm{T}$ lymphocytes from a cohort of healthy subjects. We also addressed the possibility of a link between TLR8 and viral respiratory diseases by addressing associations between the rs3761624 polymorphism and disease severity in an Argentinean population of infants hospitalized with infection by the RNA respiratory syncytial virus (RSV).

\section{Results}

Occurrence of TLR8 rs3761624 polymorphism in a healthy cohort. Blood samples were collected from healthy subjects within the National
Institute of Environmental Health Sciences (NIEHS) Environmental Polymorphisms Registry (EPR). The EPR is a resource established to facilitate genotype-driven research and translational studies of environmental diseases (15). DNA samples have been collected and stored from over 15,000 mostly North Carolinian volunteers of diverse sex, age, race, and ethnicity. DNA was genotyped for the TLR8 rs3761624 SNP (referred to as TLR8 p53RE SNP) located in the promoter region of the gene within $1 \mathrm{~kb}$ upstream of the transcription start site (TSS) (Figure 1A), along with 3 other TLR 8 coding variants: rs3764880 (Met1Val), rs5744077 (Met10Val), and rs5744082 (Arg733Pro). Demographic characteristics of the participants and genotype frequencies are summarized in Table 1 . We determined the frequencies of these TLR8 SNPs in a total of 3733 donors.

Among the individuals genotyped for the p53RE (rs3761624) A/G SNP, the A allele was more frequent, with an allele frequency 
Table 1. Demographics and distribution of TLR8 SNP allele frequencies from the EPR cohort

\begin{tabular}{|c|c|c|c|c|c|c|c|c|c|c|}
\hline \multicolumn{11}{|c|}{ EPR cohort } \\
\hline \multicolumn{11}{|l|}{ rs3761624 (p53RE) } \\
\hline Male & 1620 & 1198 & 0 & 422 & & $26.0 \%$ & 0.386 & & & \\
\hline Female & 2045 & 1122 & 746 & 117 & & $26.9 \%$ & & & & \\
\hline White & 1943 & 1256 & 372 & 315 & & $25.8 \%$ & & EUR & $27.0 \%$ & $22.7 \%$ \\
\hline Other & 194 & 79 & 58 & 57 & & $44.3 \%$ & & & & \\
\hline \multicolumn{11}{|l|}{ rs3764880 (Met1Val) } \\
\hline Global & 3733 & 2290 & 766 & 630 & 47 & $27.5 \%$ & & & $46.4 \%, n=3775$ & $28.3 \%, n=21604$ \\
\hline Male & 1633 & 1192 & 0 & 441 & & $27.0 \%$ & 0.390 & & & \\
\hline Female & 2053 & 1098 & 766 & 189 & & $27.9 \%$ & & & & \\
\hline Global & 3733 & 3419 & 212 & 91 & 21 & $5.0 \%$ & & & $3.6 \%, n=3775$ & $3.0 \%, n=21727$ \\
\hline Male & 1658 & 1598 & 0 & 60 & & $3.6 \%$ & $<0.0001$ & & & \\
\hline Female & 2054 & 1821 & 212 & 21 & & $6.2 \%$ & & & & \\
\hline White & 1961 & 1945 & 10 & 6 & & $0.6 \%$ & & EUR & $0.0 \%$ & $0.0 \%$ \\
\hline Black/African American & 1555 & 1287 & 195 & 73 & & $11.0 \%$ & & AFR & $13.1 \%$ & $11.2 \%$ \\
\hline \multirow[t]{2}{*}{ Other } & 196 & 187 & 7 & 2 & & $2.8 \%$ & & & & \\
\hline & & (GG), (G) & (GA) & $(\mathrm{AA}),(\mathrm{A})$ & & & & & & \\
\hline \multicolumn{11}{|l|}{ rs5744082 (Arg733Pro) } \\
\hline Global & 3733 & 3686 & 27 & 8 & 12 & $0.6 \%$ & & & $0.4 \%, n=3775$ & $0.4 \%, n=21637$ \\
\hline Male & 1664 & 1656 & 0 & 8 & & $0.5 \%$ & 0.271 & & & \\
\hline
\end{tabular}

of $73.5 \%$. Similarly, for the Met1Val (rs3764880) A/G polymorphism, the A allele frequency was also the most common (72.5\%). Similar and highly significant associations were found between these 2 variants, attributable to nearly perfect linkage disequilibrium $\left(r^{2}=0.955, D^{\prime}=1\right)$ between these 2 polymorphisms. The frequencies of the major allele for Met10Val (rs5744077) A/G and Arg733Pro (rs5744082) G/A variants were 95\% and 99.4\%, respectively. Because TLR 8 is located on the $\mathrm{X}$ chromosome, we performed separate frequency calculations for males and females (Table 1). No significant difference in genotype frequencies for most of the TLR8 SNPs were found between males and females in the EPR subpopulations, except for the rs 5744077 coding SNP. For the rs3761624 and rs3764880 SNPs, the minor allele frequency (MAF) in the EPR cohort (26.5\% and $27.5 \%$, respectively) was similar to that reported in the genome Aggregation Database (gnomAD, https://gnomad.broadinstitute.org/: 27.6\% and 28.3\%, respectively) but lower than reported in the 1000 Genome Project (Phase 3, http://www.1000genomes.org/: 45.2\% and $46.4 \%$, respectively). This is likely due to the low number of Asian subjects in the EPR cohort. As determined in several reports $(16,17)$, including the 1000 Genome Project and gnomAD, Asians have a higher frequency of the $G$ allele, whereas the A allele is the major allele in African and European populations. However, the MAFs of the 4 TLR 8 variants studied in the EPR cohort for the main 2 race populations (White and Black/African American) were similar to those in the 1000 Genome and gnomAD datasets (Table 1).

Activated p53 can enhance innate immune responses via a TLR8 SNP. Previously, we demonstrated in human immune cells that 


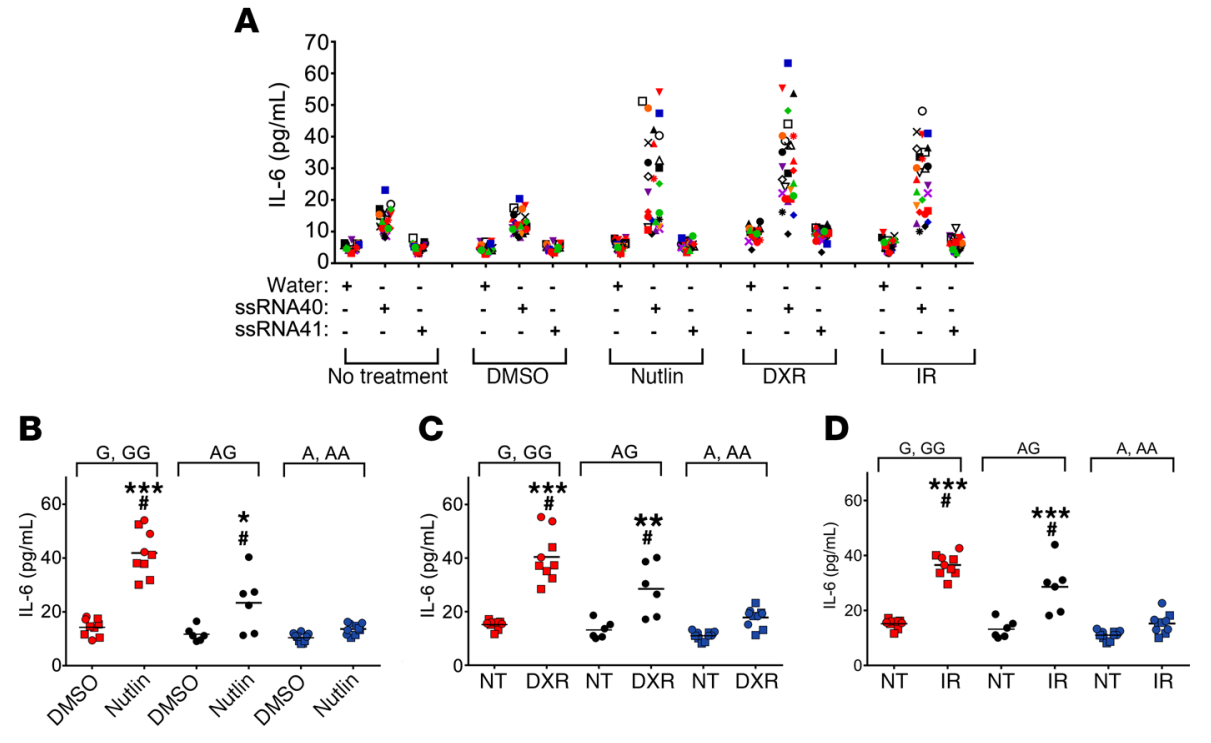

Figure 2. Activation of $\mathrm{p} 53$ by chemotherapeutic drugs amplifies the TLR8-mediated immune response in human lymphocytes in a SNP rs3761624-dependent manner. (A) IL-6 secretion measured by ELISA in blinded samples from $\mathrm{PHA}$-stimulated lymphocytes pretreated with DMSO, nutlin, DXR, or IR for 24 hours then washed twice with PBS and challenged with water, TLR8 PAMP ssRNA40 $(5 \mu \mathrm{g} / \mathrm{mL})$ or its decoy ssRNA41 $(5 \mu \mathrm{g} / \mathrm{mL})$ for 3 hours. Each dot represents a different donor $(n=27)$. Presented are IL-6 secretion profiles $(n=25)$ grouped by A/C rs3761624 genotypes after (B) nutlin, (C) DXR, and (D) IR treatments for 24 hours. ${ }^{~} P<0.001$ for drug-treated samples when compared with NT or DMSO treatments after ssRNA40 challenge for each genotype (2-tailed unpaired Student's $t$ test). ${ }^{*} P<0.01 ;{ }^{*} P<0.001 ;{ }^{* *} P<0.0001$ when compared with drug- and ssRNA40-treated group expressing the $A$ (male) or AA (female) rs3761624 SNP allele (s) (Wilcoxon signed-rank test). activation of $\mathrm{p} 53$ increases TLR8 expression in a rs3761624 SNPdependent manner (4). This led us to examine the impact of this polymorphism in p53 regulation of TLR8 expression and downstream signaling in a genotype-blinded group of 27 healthy donors (15 females and 12 males, Supplemental Table 1; supplemental material available online with this article; https://doi.org/10.1172/ JCI128626DS1). The participants were recruited from the EPR and were previously genotyped as homozygous for the A allele or $\mathrm{G}$ allele (i.e., p53 nonresponsive or responsive, respectively) or heterozygous for the rs3761624 SNP.

The existence of a SNP in a p53RE of the TLR8 promoter (AAACAT(G/A)TCA; Figure 1A) provided a unique opportunity to directly assess the relationship between p53 and TLR 8 mRNA expression. In the blinded study, we examined TLR 8 mRNA (Figure $1 \mathrm{~B}$ ) and protein expression (Figure 1C) as well as p53 occupancy in the TLR 8 promoter region (Figure 1D) in PHA-stimulated $\mathrm{T}$ lymphocytes from peripheral blood mononuclear cells that were treated ex vivo with the well-known p53 inducers nutlin, doxorubicin (DXR), and ionizing radiation (IR). Consistent with our previous report, we found significant interindividual variation in TLR8 mRNA and protein expression following nutlin, DXR, and IR treatment (Figure 1, B-D). Some subjects had highly robust TLR8 induction and p53 binding, while others had little to no TLR8 induction or p53 occupancy.

Next, we determined which SNPs were present and their relationship to $T L R 8$ induction. As shown in Figure $1, \mathrm{E}-\mathrm{G}$, induction of TLR 8 by nutlin (Figure 1E) and IR (Figure 1G), and to a lesser extent DXR (Figure 1F), was positively associated with the presence of the $G$ allele (i.e., it can bind p53). Importantly, for mRNA and protein, TLR8 induction was always high when only a $G$ allele was present (single allele in males or both alleles in females) and absent if there were only A alleles (Supplemental Figure 1). However, among the 6 female subjects who were heterozygous for these alleles, half responded poorly to both nutlin and IR. Possibly, the variation between female subjects heterozygous for the SNP is related to X chromosome inactivation, as discussed below.
Similar patterns of TLR8 mRNA expression after p53activating drug treatments were observed when the current data were combined with our previous population study that included human $\mathrm{T}$ lymphocytes and alveolar macrophages from randomly selected healthy donors, providing a total of 49 subjects (Supplemental Table 2). The combined results demonstrate that the TLR8 gene expression-genotype association after p53 activation by DNA-damaging drugs in immune primary human cells is strongly associated with the presence of the G (or GG in females) allele in the p53RE of the TLR8 promoter (Supplemental Figure 2). There were no apparent differences between males and females in the $G$ versus A or GG versus AA allele frequencies.

Using linear regression, we addressed the relationship between TLR8 mRNA and protein expression (fold-change) and rs3761624 genotype from the lymphocytes treated with the 3 p53-activating agents (Supplemental Figure 3). TLR8 mRNA and protein levels were significantly correlated with rs3761624 genotype, consistent with the p53-responsive $\mathrm{G}$ allele conferring enhanced expression in response to p53 activation. Representative examples for TLR8 protein expression profiles from different rs3761624 SNP genotypes are presented in Supplemental Figure 4 , showing that in comparison with the common allele (A or AA) the GG genotype correlates with a high induction of TLR8 protein appearance in response to p53 activating drugs. The $G$ allele also strongly correlated with the ability of p53 to bind the p53RE in the promoter region of TLR 8 after nutlin, IR, and DXR treatments (Supplemental Figures 3 and 5). Furthermore, pretreatment with the $\mathrm{p} 53$ inhibitor pifithrin-alpha repressed the TLR 8 expression in an allele-specific manner, confirming the SNP-dependent role of p53 in TLR8 expression (Supplemental Figure 6) and showing that the human TLR8 is an important component in the p53/immune axis. As expected, while expression of the p53 target gene p21 was affected by pifithrin-alpha, the expression was not influenced by the TLR8 p53RE SNP.

p53 mutants can induce TLR8 via rs3761624 TLR8 SNP. We established that WT p53 as well as some tumor-associated p53 mutants can modify expression of several TLR genes (18). To 
A

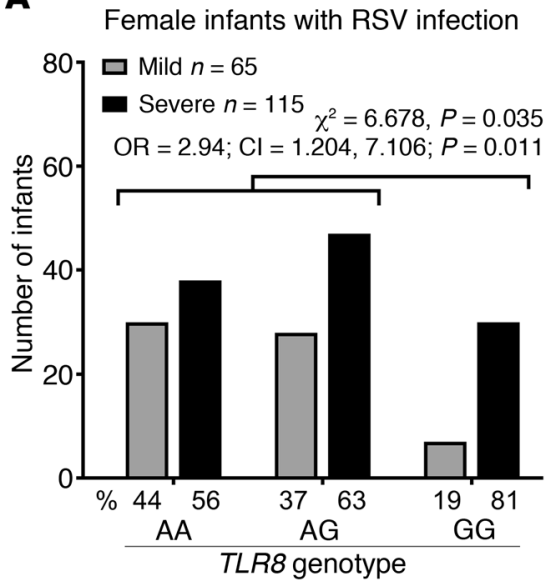

B

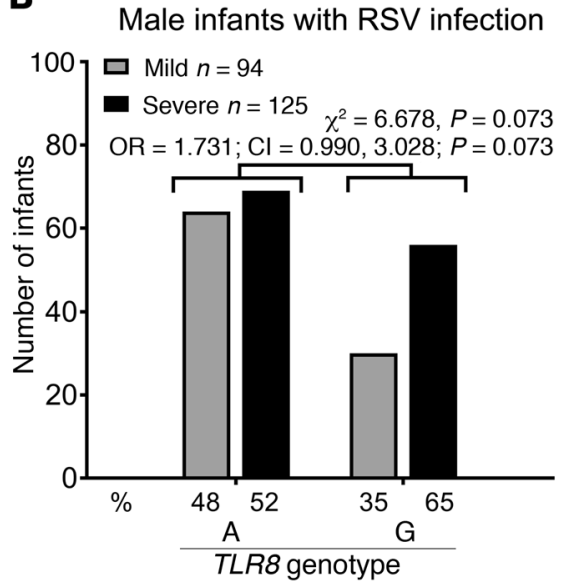

Figure 3. TLR8 rs3761624 polymorphism contributes to severity of RSV infection in infants. DNA was isolated from whole blood, and infants with mild or severe RSV infection were genotyped for TLR8 rs3761624 SNP. $\chi^{2}$ analysis of RSV disease severity among (A) female and (B) male homozygous WT, heterozygous, and homozygous variants was used to determine association of genotype with increased disease severity. The percentage of patients for each genotype is shown below each bar. assess the potential of the TLR 8 rs 3761624 variant to respond to transactivation by cancer-associated p53 mutants, the p53RE of the TLR8 promoter containing either the A or G SNP was cloned into a pGL3 promoter luciferase reporter plasmid. A total of 10 p53 mutants were cotransfected along with the reporter plasmid in p53-null SaOS2 human cells. We first confirmed that WT p53 drives the transcription of the luciferase construct containing the minor G allele (Supplemental Figure 7). The p53 mutants A138V, $\mathrm{P} 151 \mathrm{H}, \mathrm{H} 178 \mathrm{Y}$, and $\mathrm{R} 337 \mathrm{H}$ also induced transactivation of TLR8 for the p53RE G allele. The M133T, P190L, and R337C p53 mutants were unable to induce transactivation. The p53 hotspot mutants R175H, R273H, and R280K, which are completely deficient for p53 transactivation, also lacked transactivation from the reporter. Overall, we found that some p53 tumor-associated mutants that retain transactivation abilities can drive $T L R 8$ expression in a SNP-dependent manner.

p53 enhances TLR8-mediated immune response via a TLR8 rs3761624 SNP. To determine if the SNP-dependent regulation of TLR8 expression by 553 has biological consequences, the PHA-stimulated lymphocytes from 25 donors were pretreated with a panel of p53-activating drugs for 24 hours and washed twice with saline buffer. They were then exposed to the TLR8 agonist ssRNA40 or control ssRNA41 (uridine replaced by adenosine) for 3 hours and the appearance of the proinflammatory cytokine interleukin 6 (IL-6) was determined in the blinded samples (Figure 2A). More than half the samples that were pretreated with nutlin, DXR, or IR had significant increases in IL-6 in response to ssRNA40, unlike the ssRNA41-exposed, nontreated, or DMSO-treated cells. Grouping the blinded samples according to genotype, we found that the p53-activating drugs led to higher production of IL- 6 after stimulation by the ssRNA40 in a p53RE-SNP-dependent manner (Figure 2, B-D). A 2- to 3-fold greater IL-6 production was found in females homozygous for the $G$ allele and males with the $G$ allele compared with homozygous donors for the common A allele. These results confirm that activation of $\mathrm{p} 53$ amplifies the TLR8-mediated immune response in human lymphocytes in a SNP rs3761624-dependent manner. As previously found for the expression and p53 binding analysis, wide interindividual variation was found in responses in cells from heterozygous female donors.
Role of TLR8 rs3761624 in RSV disease severity. Given the role that TLR8 plays in innate immune responses against pathogens, mainly RNA viruses, we sought to determine the clinical impact of TLR 8 rs3761624. RSV is the primary cause of lower respiratory tract infection and bronchiolitis during infancy and childhood (19). It causes activation of innate immune responses by triggering TLR pathways, resulting in elevated cytokines and type I interferon levels (20). We hypothesized that the TLR8 rs3761624 SNP would associate with susceptibility to severe human RSV disease in infected infants. RSV disease severity was based on the need for oxygen supplementation and consequent hospitalization (21-23). To address the potential role of the TLR8 p53RE SNP, we evaluated RSV disease severity in a previously described prospective case-control study that evaluated treatments of infants ( $<9$ months of age, $n=$ 399) (21-23) who visited the hospital with RSV-associated bronchiolitis. They were considered to have severe disease $(n=240)$ if their oxygen saturation level upon enrollment was less than $93 \%$ in room air, while infants with greater than $93 \%$ blood $\mathrm{O}_{2}$ saturation were classified as having mild disease $(n=159)$.

We genotyped the RSV-infected infants for the TLR8 p53RE SNP status and found that the combined allelic frequencies were similar to those reported in the 1000 Genome Project (MAF $=$ $40.3 \%$ vs $45.2 \%$ ). Because TLR 8 is X-linked, we analyzed the RSV disease severity risk verus TLR 8 SNP by sexual phenotype. We found a significant increase for severe RSV disease among female and male infants who also had the p53-responsive $G$ allele. Female infants with the GG allele were at significantly greater risk of developing severe RSV disease $\left(\chi^{2}=6.678 ; P=0.035\right.$; OR $=$ $2.924 ; 95 \% \mathrm{CI}=1.204,7.106 ; P=0.011)$ when compared with the homozygous major A and heterozygous variant (Figure 3A). Similarly, for the male infants (Figure 3B), the presence of the minor $\mathrm{G}$ allele was significantly associated with disease severity $\left(\chi^{2}=6.678\right.$; $P=0.073 ; \mathrm{OR}=1.731 ; 95 \% \mathrm{CI}=0.990,3.028 ; P=0.071)$. Thus, the rs3761624 TLR8 polymorphism is a potential prognostic biomarker for predicting disease severity in response to RSV infection. Using multiple logistic regression, we found the TLR8 $\mathrm{p} 53 \mathrm{RE}$ SNP was associated with increased risk of RSV disease in this population, and the significance did not change after adjusting for sexual phenotype, socioeconomic status, and breastfeeding (data not shown). Consistent with the above results, logistic regression 
analysis also revealed an association between males carrying the $\mathrm{G}$ allele and females carrying the GG allele and longer duration of hospitalization in days $(\mathrm{OR}=1.90,95 \% \mathrm{CI}=1.02,3.52)$ (Supplemental Table 3). We also performed a logistic regression analysis using as outcome severity based on hospitalization (yes or no) and comparing 2 variables: the TLR 8 p53RE SNP genotype and age in months (continuous variable). The TLR 8 genotype as well as age independently associated with hospitalization due to RSV lower respiratory tract infection (LRTI). Hospitalization was determined by $\mathrm{O}_{2}$ saturations less than $93 \%$. We found that there was a $1.5-$ fold greater chance of being hospitalized if a male or female had TLR8 p53RE G/GG allele (OR = 1.50, 95\% CI $=1.06,2.12)$, and a protective effect for older age $(\mathrm{OR}=0.86,95 \% \mathrm{CI}=0.81,0.92)$ (Supplemental Table 4).

\section{Discussion}

Variation between individuals and populations in immune activities can modulate susceptibility to infectious and autoimmune diseases $(21,24)$. SNPs located in noncoding regions such as promoters and other transcriptional regulatory regions may be important host genetic factors that contribute to susceptibility to many common immune-related diseases $(25,26)$. For example, immune responses to pathogens between African and European populations were associated with allele variation in SNPs in regulatory regions (27). Since regulatory genetic variants could act as modifiers of disease risk, their identification is important especially for the molecular characterization of complex traits. Variants in regulatory sequences represent an important class of genetic variation in complex traits and disease risk (28). The variants can result in network plasticity with the possibility of fine-tuning overall gene expression programs responding to environmental challenges, as reported in studies with WT and mutant p53 (29). The present study suggests that variation in targeted p53RE sequences may contribute to 553 transactivation specificity in response to stress signals, which in turn could result in biological diversity of a population. Here, we demonstrated the functional effects of TLR 8 promoter rs3761624 variants on TLR $8 \mathrm{mRNA}$, protein expression, and cytokine production that are induced ex vivo by activated p53 in primary $\mathrm{T}$ lymphocytes from healthy subjects following exposure to TLR8-specific agonists.

A critical component of innate immunity is detection of a wide range of pattern recognition molecules by different immune sensors, including the TLRs. Polymorphisms in TLR genes can impact how affected individuals respond appropriately to TLR ligands, leading to an altered susceptibility and the development of pathogenic disease $(21,30)$. During the evolution of the 10 TLRs in the innate immune response, the endosomal TLRs $-3,-7,-8$, and -9 evolved under the strongest purifying selection, indicating their essential role in host survival. Interestingly, these 4 TLRs correspond to those receptors known to recognize nucleic acids and are involved primarily in the recognition of viruses (31). While there have been association studies between TLR8 polymorphisms and pathogen infections and other diseases (for a review, see ref. 32), understanding of the functional effects of these TLR8 genetic polymorphisms is limited.

Our study highlights the relevance of TLR8 in viral infections and provides what we believe is the first report on a genetic $T L R 8$ variant associated with severity of RSV disease. RSV is a single-stranded RNA virus of the Paramyxoviridae family that may cause acute bronchiolitis and is commonly associated with lower respiratory tract infections in infants and young children. Around 34 million cases of RSV lower respiratory tract infection of young children occur annually, of which approximately $10 \%$ require hospitalization. The clinical course of RSV infection is highly variable, which might be due in part to genetic variations in immune pathway genes. Population studies in RSV pathogenesis have demonstrated associations of severe RSV in healthy infants with genetic polymorphisms in genes involved in the regulation of the immune and proinflammatory response including several cell-surface adhesion molecules, antiviral proteins, cytokines, and pathogen receptors $(22,33)$. Among TLRs, interactions between RSV and TLR variants have been described, mainly for TLR3 and TLR4 (21, 34-36). Unfortunately, due to chromosome X linkage, TLR8 SNPs have been largely excluded from RSV population studies (37). An exception to this concept was identified in a German male population (38) where a weak association was found between TLR8 rs2407992 and severity of RSV infections.

In the present study, we used a population case-control study to test the hypothesis that the presence of the p53-responsive $G$ allele of the TLR8 rs3761624 SNP is associated with increased severity of RSV determined illness in females as well as in males. The results for the males were consistent with those of the females, albeit the $P$ value is 0.07 while for females it is 0.01 . We hypothesize that for the TLR 8 sex-linked gene there is a dosage compensation effect such that one copy per diploid genome is less deleterious than 2 copies of the 533 responsive TLR8. Our results were consistent with the hypothesis that higher TLR8 expression induced by p53 leads to greater downstream cytokine production, causing increased lung disturbance. It would be interesting to examine the effect of mutant p53 alleles on cytokine profiles in response to TLR8 natural activators such as ssRNA viruses. During RSV replication, TLR 8 mRNA appears upregulated in RSV infected human tracheal epithelial cells (39), as well as in cells from nasopharyngeal washes collected from RSV-infected infants compared with noninfected individuals (40). Contrary to those studies, Bendelja and collaborators reported decreased TLR8 protein expression levels in the monocytes of a small population ( $n=15)$ of infants who were simply identified as having acute RSV infection (41). It is not clear how those findings relate to those here in which we address the genetically based differences in severity of response in a much larger population where it is possible to relate to a specific difference in a functional SNP. Because of the $\mathrm{X}$-linked nature of the SNP, we identified sexual phenotype differences. In the monocyte study, sex was not specified. Thus, it would be interesting in future large studies such as those described here to determine TLR8 levels in lymphocytes as well as monocytes and compare across severity of infection, sexual phenotype, and TLR8-p53-SNP allele genotypes. Overall, our data suggest that different TLR 8 haplotypes affect RSV viral replication patterns in immune cells and that consideration of TLR variants will increase our understanding of RSV disease progression. Considering the limitations in the size of our current study, further studies that apply a similar approach using another population with different backgrounds as well as including cohorts of older populations which are also RSV sensitive will be useful. 
Because p53 can influence TLR8 expression depending on the presence of the TLR8 rs3761624 SNP G allele, induction of the stress-responsive TP53 gene by RSV itself might be expected to contribute to TLR8-associated responses. However, there have been considerable differences in reports of p53 induction, from reduced p53 transcription and p53 protein levels to the opposite (42-45). This could be due at least in part to the systems, cell lines and/or proteins of the RSV virus that have been used. In support of our observations, a recent study suggests that RSV infection induces DNA damage in a human cell line model and consequently affects p53 stabilization (45).

As we show, there is a strong p53-responsive TLR8 SNPdependent effect in the disease severity. Formally, the effect could be due to associated infections from other agents that are possibly a TLR8 immune response. For example, RSV-disease severity is often strongly associated with viral and bacterial coinfections. In fact, infants were tested in our study for RSV, rhinoviruses, hPIV14 , influenza virus $\mathrm{A}$ and $\mathrm{B}$, and human metapneumovirus, and all participating subjects were infected only with RSV (23). Because TLR8 is a major sensor of bacteria in human primary monocytes and macrophages (46), it will be important to consider its impact on the correlation between the RSV-disease severity and TLR8 rs3761624 SNP in further studies.

In the EPR cohort as well as other population databases (e.g., 1000genomes, gnomAD), the rs3761624 variant was found to be in strong linkage disequilibrium with a nonsynonymous polymorphism located at exon 1 of TLR8 (rs3764880), which previously was identified as a functional polymorphism associated with several pathogen infections $(47,48)$. The rs 3764880 change from A to $\mathrm{G}$ that modifies the start codon of one of the TLR 8 isoforms (transcript variant 2 [TLR8v2]), results in a truncated TLR8 isoform with a shorter signal peptide than the common allele (49). This SNP increases TLR8 expression, influences NF- $\kappa \mathrm{B}$ activation, and has been associated with modulation of cytokine induction (11). Although we did not evaluate expression of the TLR8 isoforms, we hypothesize that $\mathrm{p} 53$ will influence the levels of expression of $T L R 8$ via the rs3761624 variant while the rs3764880 SNP impacts directly TLR8-mediated immune response. In agreement with this hypothesis, several population studies have found that the rs3761624-rs3764880 G-G genotype confers resistance and better immune response against pathogen infection. Interestingly, the rs3764880 G allele is also differentially associated with HIV status in distinct human populations $(49,50)$. In addition, this allele protects against tissue damage in active tuberculosis $(51,52)$.

Unlike for cells from the G and A allele homozygous TLR 8 donors, we found substantial variation in p53 stress-induced TLR8 responses in heterozygous cells. We speculate that such variation could be due in part to the random $\mathrm{X}$ chromosome inactivation process in mammals, potentially resulting in donors that primarily express only the $\mathrm{G}$ or A allele. Interestingly, in females a fraction of $\mathrm{X}$ chromosome genes escape such inactivation, resulting in genes having a biallelic expression and a double gene dosage. This situation can lead to sex bias in gene expression (53). At least 50 protein-coding genes associated with immune responses are located on the $\mathrm{X}$ chromosome in addition to TLR8. Included are TLR7, BTK4, IKBKG, and IRAK1, which have been found to have biallelic expression (54). Possi- bly, higher expression of these genes in females compared with males may confer an advantage in responding to and resolving acute infections, but they could also be more prone to certain diseases including some autoimmune disorders (55). The mechanisms of $\mathrm{X}$ chromosome inactivation and associated gene escape are still poorly understood (53). Whether or not TLR 8 has biallelic features remains to be determined.

Information on the in vivo function of TLR8 in terms of downstream signaling and responses to agonists is limited, in part due to phylogenetic differences in experimental models. Though mouse and human TLR8 proteins are highly related, there appear to be substantial differences in receptor specificity as well as natural/physiological and synthetic TLR ligands (56). Moreover, we reported that TLR8 was not $\mathrm{p} 53$ responsive in mouse immune and epithelial cells due to the TLR 8 promoter regulatory region lacking a functional p53RE (4). In addition, we found that human cancer cell lines lacked TLR8 expression $(13,57)$ so that the potential role of TLR8 in cancer as well as its crosstalk with p53 could not be addressed. Our findings with TLR8 expression in immune cells are expected to provide opportunities for studying the TLR8 network, its immune-associated diseases and anticancer therapy.

Our findings are also relevant to cancer development and therapies. In light of recent approaches that utilize the host immune system to eliminate tumor cells and establish a long-term tumor cell surveillance, it may be possible to employ features of TLR8 signaling in therapies. TLR8 stimulates the production of several inflammatory mediators including Th-1-polarizing cytokines and the functional enhancement of several immune effector cells including monocytes, myeloid dendritic cells, and natural killer cells $(8,58)$. Recently, Dietsch et al. (59) concluded that patients with late-stage cancer showed a robust immune activation in response to TLR8 activation by motolimod (VTX2337), a potent and selective TLR8 agonist. Specific subsets of T cells are also known to selectively express TLRs. Tregs maintain peripheral tolerance, a state of unresponsiveness of the immune system to ensure that self-reactive $\mathrm{T}$ and $\mathrm{B}$ cells do not cause autoimmune disease and play a critical role in control of the immune response in infection, tumor defense, organ transplantation, and allergy. TLR8 appears to modulate the suppressive activity of naturally occurring Tregs. Peng et al. (60) found that TLR8 is expressed in human Tregs and that the corresponding ligands ssRNA40 and ssRNA33 reverted the suppressive function of Tregs, leading to enhanced $\mathrm{CD} 4^{+} \mathrm{T}$ cell proliferation.

Our findings that DNA damage-induced p53 can influence TLR8 expression and downstream signaling in a SNPdependent manner could be clinically relevant for immunebased cancer therapies. Since the TLR8 agonist ssRNA40 triggers IL-6 production in a p53RE-SNP-dependent manner in lymphocytes, p53 -dependent activation of TLR8 (depending on the rs3761624 SNP) in combination with TLR8 agonist might be employed to enhance tumor-directed immune responses as used for checkpoint blockade immunotherapy. For example, this combination might promote the activation of $\mathrm{CD}^{+} \mathrm{T}$ cells and their ability to effectively kill tumor cells. Thus, the suppressive function of Tregs could be augmented or attenuated depending on the nature of TLR8 stimulation via p53 activation, which has implications in immunotherapy of can- 
cers, infections, and autoimmunity. We note that many cancer treatments involve activation of p53 in either the tumor or the surrounding tissue.

In extending our p53/immune axis findings to a variety of cancer-associated p53 mutants, we found that p53 mutants with altered transcriptional function had correspondingly altered responses to TLR ligands and subsequent appearance of cytokines. For example, p53 directly increases TLR3 and TLR5 transcription. Cancer-associated p53 mutants differed in their downstream responses to the TLR3 ligand (poly(I:C)) (18), and they could alter TLR5 responses in cancer cells (61). Here, we found that the A138V, P151H, H178Y, and R337H p53 mutants could modulate TLR8 expression in a SNP-dependent manner. Interestingly, this same group of mutants also retained the ability to regulate TLR3 (18) and TLR5 (61) expression. Another group of mutants that included M133T, P190L, and R337C did not impact $T L R 8$ expression, although they have been shown to have different transactivation abilities toward other TLR genes (18). Although the mutants we examined retain function, they can exhibit change-of-spectrum behavior in terms of target sequences activated and levels of activation (29). Our results suggest that knowledge of the p53 mutation status in tumor cells could help inform patient responsiveness to immunotherapies that also involve chemotherapeutic agents.

Overall, our findings demonstrate that rs3761624 may play an important role in TLR8 biology by mediating TLR8 immune responses. Knowledge of the p53-responsive rs3761624 SNP can influence diagnosis and prognosis of RSV severity and potentially other TLR8-associated diseases. Furthermore, our results are expected to be relevant to other viral and pathogen-associated diseases such as Zika, HIV, and TB, where TLR8 has been implicated in their recognition as well as in the initiation and mediation of inflammatory cytokine responses $(62,63)$.

\section{Methods}

Human subjects. Thirty healthy subjects (24-69 years) were recruited from the EPR under the approved National Institute of Environmental Health Sciences (NIEHS) Institutional Review Board Protocol 04-E-0053. The EPR is a long-term project to facilitate genotype-driven research and translational studies of environmental diseases (15). DNA samples have been collected and stored from over 15,000 volunteers from a racially diverse population including many in the greater North Carolina Triangle Region, USA (15). Each subject signed an informed consent form after a thorough explanation of the study. Screening procedures consisted of a medical history, which included diagnosed medical conditions and medication history, followed by physical examination including blood pressure, weight, height, and blood draw.

Allelic discrimination genotyping for TLR8 SNPs. Genotyping was performed by the NIEHS Molecular Genomics Core Laboratory. Briefly, genomic DNA was prepared from human blood samples from the EPR cohort using the Qiagen Autopure LS system. DNA samples were genotyped for the TLR8 rs3761624 SNP that created a responsive p53RE as well as the coding TLR8 SNPs (rs3764880, rs5744077, and rs5744082), using the Illumina customized GoldenGate Assay. A subset of samples was genotyped for the TLR8 rs rs3761624 SNP using the TaqMan SNP genotyping assay C_27497635_10 by the EPR laboratory.
Human RSV disease population study. A prospective case-control study was conducted in Buenos Aires, Argentina, between 2003 and 2006. The infantswere recruited from the following hospitals: Hospital Francés, Hospital Nacional Dr. Alejandro Posadas, Hospital Evita Pueblo de Berazategui, and Hospital Mi Pueblo de Florencio Varela. Demographic information as well as inclusion/exclusion selection criteria for RSV severity and control population were described in previous studies $(21,22)$. Briefly, invited participants were previously healthy full-term infants younger than 1 year of age and born after September 15 of the previous year (15 days after the end of RSV season in Buenos Aires), with signs and symptoms of bronchiolitis for the first time in their lives. Infants with bronchiolitis were recruited as cases when their oxygen saturation on enrollment was lower than $93 \%$ in room air. Genotyping of these infants was performed using nasal aspirates.

Cell culture. Human lymphocytes were isolated and cultured from the blood of healthy donors as previously described (4), in accordance with a NIEHS IRB-approved protocol 04-E-0053. For T cell stimulation, cells were activated with phytohemagglutinin $\mathrm{M}$ form (PHA-M, $1.5 \% \mathrm{vol} / \mathrm{vol}$, Invitrogen) for 72 hours. Cells were treated starting 48 hours after PHA addition, and cell cultures were harvested 24 hours later. Cells were plated 18-24 hours before being treated with nutlin-3 $(10 \mu \mathrm{M})$ and DXR (1 $\mu \mathrm{M}$, MilliporeSigma); treatment was for 24 hours unless otherwise indicated. DMSO (0.1\% volume, MilliporeSigma) was used as solvent. For IR treatment, cells were irradiated at $1.56 \mathrm{~Gy} / \mathrm{min}$ from a Shepherd cesium irradiator in culture medium at room temperature. Where indicated, cells were pretreated with $30 \mu \mathrm{M}$ pifithrin- $\alpha$ (MilliporeSigma) for 3 hours, prior to p53 reactivation or drug treatment. The SaOS2 (HTB-85) cell line was purchased from the American Type Culture Collection (ATCC) and cultured as indicated by ATCC. All cell cultures were maintained at $37^{\circ} \mathrm{C}$ with $5 \% \mathrm{CO}_{2}$.

Plasmids, transfection, and luciferase reporter assay. WT p53 expression vector pC53-SN3 was a gift from B. Vogelstein (Johns Hopkins University, Baltimore, MD). All the p53 point-mutant constructs described in the text were derived from pC53-SN3 vector and prepared using the QuikChange II XL site-directed mutagenesis kit (Stratagene) according to the manufacturer's instructions. Luciferase reporter constructs for TLR8 p53RE-SNP containing the p53RE-A or p53RE-G alleles were constructed in pGL3-promoter vectors (Promega) double digested by XhoI and KpnI using a pair of complementary oligonucleotides generating compatible cloning ends. Sequences of all the constructs developed for this study were confirmed by DNA sequencing. Details on primer sequences are described in the Supplemental Material. In all transient transfection experiments, pRLV40 containing the Renilla luciferase cDNA (Promega) was used to normalize for transfection efficiency. Transient transfections with p53 mutants were carried out using FuGENE 6 reagent (Promega), according to the manufacturer's instructions. For luciferase assays, $p 53^{--}$SaOS2 cells seeded into 12-well plates were transfected with $0.25 \mu \mathrm{g}$ of the luciferase reporter vector together with 50 ng p53 expression vector; 25 ng pRL-TK Renilla luciferase plasmid (Promega) was used as a transfection efficiency control. Luciferase activities were measured 48 hours after transfection with a Synergy2 Multi-mode Microplate reader (Bio-Tek Instruments Inc.) using the dual-luciferase assay system (Promega), according to the manufacturer's protocol. Relative luciferase activity for each construct was defined as the mean value of the firefly luciferase/Renilla luciferase ratios obtained from 3 independent experiments, each performed in triplicate. 
Real-time RT-PCR analysis. Total RNA was extracted from lymphocytes in the presence of DNase using RNeasy mini kit (Qiagen), according to the manufacturer's instructions. RNA concentration was measured by Nanodrop (Thermo Fisher Scientific). The cDNA was synthesized using TaqMan reverse transcription reagents and random hexamers (Applied Biosystems). The same amount of cDNA was used to analyze TLR8, GUSB, and GAPDH in triplicate. Real-time Taqman PCR was run on an ABI 7900HT instrument using primer and probes from Applied Biosystems (see Supplemental Material). The level of target gene expression was normalized to GUSB using the $\triangle \mathrm{Ct}$ method.

IL-6 measurement by ELISA. After cell stimulation, supernatants were collected, centrifuged to remove cellular debris, and assayed immediately or stored at $-80^{\circ} \mathrm{C}$ until assayed. Secreted IL- 6 concentration in cell-free supernatants from treated cells was measured using the IL-6 Human ELISA Kit (catalog BMS213HS, Invitrogen), following the manufacturer's protocol. Absorbance $(450 \mathrm{~nm})$ was measured on a Biotek Synergy 2 plate reader. Detection limits of IL-6 ELISA were $8 \mathrm{pg} / \mu \mathrm{L}$. All measurements were performed in duplicate, and mean values of the 2 measurements were used for statistical analysis.

Chromatin immunoprecipitation assay (ChIP). After treatment, the cellular material was cross-linked with methanol free $1 \%$ formaldehyde (MilliporeSigma). Then, cell lysates were sonicated using conditions that yield 200-500 bp DNA fragments using a Bioruptor XL (Diagenode). DNA-protein complexes were immunoprecipitated with $1 \mu \mathrm{g}$ DO-1 p53-specific monoclonal antibody per condition. Mouse Ig (Santa Cruz Biotechnology, sc-126) was used as a negative control. Quantitative PCR (qPCR) was performed on immunoprecipitated chromatin to determine p53 enrichment occupancy on TLR8 and $p 21$ promoter regions. Amplification of $G A D P H$ promoter region was used as a negative control. ChIP primers for TLR8, $p 21$, and GADPH were previously described (4, 18). qPCR and melting curve analysis were performed using the SYBR Green (Invitrogen) dye detection method on the ABI PRISM 7900 HT Sequence Detection System under default conditions. Enrichment in the ChIP samples was calculated as fraction of the input (\%).

TLR8 protein expression analysis by flow cytometry. Direct intracellular staining was performed using a conjugated TLR8 antibody (44C143) (Alexa Fluor 647; Novus) to evaluate changes in TLR8 protein expression. Briefly, $1 \times 10^{6}$ cells from each culture were harvested and washed in FACS buffer (PBS+0.5\% BSA+0.01\% sodium azide), pelleted and suspended in permeabilization/fixation buffer (catalog 00-5333-54, Thermo Fisher Scientific), and left for 20 minutes at room temperature. The cells were then washed in permeabilization buffer, pelleted, and stained with $2 \mu \mathrm{g}$ anti-TLR8-Alexa Fluor 647 at $4^{\circ} \mathrm{C}$ for 1 hour. IgG1/ $\kappa$ isotype primary conjugated Alexa Fluor 647 antibody (catalog DDXCH01A647, Novus) served as a negative control. Samples were prepared and analyzed in duplicate, and a mini- mum of 10,000 cells was counted for each sample. Finally, the samples were washed, suspended in FACS buffer, and analyzed using a LSR II flow cytometer (BD Biosciences) for TLR8 detection. Captured data of gated populations were analyzed using FloJo software.

Statistics. Statistical analysis was performed using GraphPad Prism, version 6 for Windows software. Differences between the treatment groups were analyzed using the 2-tailed unpaired Student's $t$ test or the Wilcoxon signed-rank test for matched pair analysis when normal distribution could not be assumed. A value of $P$ less than 0.05 was considered statistically significant. For the RSV population study, genotype and allele frequencies for TLR 8 were calculated through $\chi^{2}$ test, and the Pearson's $\chi^{2}$ test was used to evaluate the Hardy-Weinberg equilibriums of the genotype distribution. Multiple logistic regression analysis (SigmaPlot 13.0, SYSTAT) was performed to evaluate an association between TLR8 rs3761624 alleles and RSV severity infection and age. The $P$ values, ORs, and 95\% CIs were calculated.

Study approval. The IRBs of both the French Hospital (Buenos Aires) and the network of Hospital Materno Infantil de San Isidro (which encompasses the 3 hospitals in the northeastern region of Buenos Aires), as well as the IRB of Johns Hopkins University (Baltimore, MD, USA), approved the protocol for the human RSV disease population study.

\section{Author contributions}

DM, FPP, SRK, and MAR conceived and designed the experiments. DM, JS, JM, MTC, and CLI performed the experiments. DM, SHS, CLI, and SRK analyzed the data. DM, MTC, FPP, SHS, and SRK contributed reagents/materials/analysis tools. DM, SKR, and MAR wrote the paper.

\section{Acknowledgments}

We are grateful to the anonymous donors for providing the samples used in this study. We thank the members of the National Institute of Environmental Health Sciences (NIEHS) Clinical Research Branch (CRB), with special thanks to the staff of the CRU and EPR laboratories. We thank Carl Anderson and Stavros Garantziotis for critical review and comments. This work was supported by the Intramural Research Program of the NIH, NIEHS (project Z01ES065079 to MAR, and project Z01-ES100557 to SRK).

Address correspondence to: Daniel Menendez or Michael A. Resnick, 111 TW Alexander Drive, Rall Building Room D338 (DM), Rall Building Room D342 (MAR), Research Triangle Park, North Carolina 27709, USA. Phone: 984.428.4105; Email: menendez@ niehs.nih.gov (DM). Phone: 984.287.4109; Email: resnick@niehs. nih.gov (MAR).
1. Wu J, Chen ZJ. Innate immune sensing and signaling of cytosolic nucleic acids. Annu Rev Immunol. 2014;32:461-488.

2. Zuniga EI, Macal M, Lewis GM, Harker JA. Innate and adaptive immune regulation during chronic viral infections. Annu Rev Virol. 2015;2(1):573-597.

3. Takaoka A, et al. Integration of interferonalpha/beta signalling to 553 responses in tumour suppression and antiviral defence. Nature. 2003;424(6948):516-523.

4. Menendez D, Shatz M, Azzam K, Garantziotis S,
Fessler MB, Resnick MA. The Toll-like receptor gene family is integrated into human DNA damage and 553 networks. PLoS Genet. 2011;7(3):e1001360.

5. Menendez D, Nguyen TA, Snipe J, Resnick MA. The cytidine deaminase APOBEC3 family is subject to transcriptional regulation by p53. Mol Cancer Res. 2017;15(6):735-743.

6. Muñoz-Fontela C, Mandinova A, Aaronson SA, Lee SW. Emerging roles of $\mathrm{p} 53$ and other tumour-suppressor genes in immune regulation.
Nat Rev Immunol. 2016;16(12):741-750.

7. Nguyen TT, et al. Revealing a human p53 universe. Nucleic Acids Res. 2018;46(16):8153-8167.

8. Kawai T, Akira S. The role of pattern-recognition receptors in innate immunity: update on Toll-like receptors. Nat Immunol. 2010;11(5):373-384.

9. Iwasaki A, Medzhitov R. Toll-like receptor control of the adaptive immune responses. Nat Immunol. 2004;5(10):987-995.

10. Han X, et al. Epigenetic regulation of tumor necrosis factor $\alpha(\mathrm{TNF} \alpha)$ release in human mac- 
rophages by HIV-1 single-stranded RNA (ssRNA) is dependent on TLR8 signaling. J Biol Chem. 2012;287(17):13778-13786.

11. Gantier MP, et al. Genetic modulation of TLR8 response following bacterial phagocytosis. Hum Mutat. 2010;31(9):1069-1079.

12. Johnson TP, Tyagi R, Patel K, Schiess N, Calabresi PA, Nath A. Impaired toll-like receptor 8 signaling in multiple sclerosis. J Neuroinflammation. 2013;10:74.

13. Tomso DJ, et al. Functionally distinct polymorphic sequences in the human genome that are targets for p53 transactivation. Proc Natl Acad Sci U S A . 2005;102(18):6431-6436.

14. Menendez D, Inga A, Resnick MA. The expanding universe of p53 targets. Nat Rev Cancer. 2009;9(10):724-737.

15. Chulada PC, Vainorius E, Garantziotis S, Burch LH, Blackshear PJ, Zeldin DC. The Environmental Polymorphism Registry: a unique resource that facilitates translational research of environmental disease. Environ Health Perspect. 2011;119(11):1523-1527.

16. Salie M, et al. Association of toll-like receptors with susceptibility to tuberculosis suggests sex-specific effects of TLR8 polymorphisms. Infect Genet Evol. 2015;34:221-229.

17. Sun Q, Zhang Q, Xiao HP, Bai C. Toll-like receptor polymorphisms and tuberculosis susceptibility: A comprehensive meta-analysis. J Huazhong Univ Sci Technol Med Sci. 2015;35(2):157-168.

18. Menendez D, Lowe JM, Snipe J, Resnick MA. Ligand dependent restoration of human TLR3 signaling and death in 553 mutant cells. Oncotarget. 2016;7(38):61630-61642.

19. Shi T, et al. Risk factors for respiratory syncytial virus associated with acute lower respiratory infection in children under five years: Systematic review and meta-analysis. J Glob Health. 2015;5(2):020416.

20. Borchers AT, Chang C, Gershwin ME, Gershwin LJ. Respiratory syncytial virus--a comprehensive review. Clin Rev Allergy Immunol. 2013;45(3):331-379.

21. Caballero MT, et al. TLR4 genotype and environmental LPS mediate RSV bronchiolitis through Th2 polarization. JClin Invest. 2015;125(2):571-582.

22. Ciencewicki JM, et al. A genetic model of differential susceptibility to human respiratory syncytial virus (RSV) infection. FASEB J. 2014;28(4):1947-1956.

23. High M, et al. Determinants of host susceptibility to murine respiratory syncytial virus (RSV) disease identify a role for the innate immunity scavenger receptor MARCO gene in human infants. EBioMedicine. 2016;11:73-84.

24. Fairfax BP, Knight JC. Genetics of gene expression in immunity to infection. Curr Opin Immunol. 2014;30:63-71.

25. Hindorff LA, et al. Potential etiologic and functional implications of genome-wide association loci for human diseases and traits. Proc Natl Acad Sci U S A. 2009;106(23):9362-9367.

26. Schaub MA, Boyle AP, Kundaje A, Batzoglou S, Snyder M. Linking disease associations with regulatory information in the human genome. Genome Res. 2012;22(9):1748-1759.

27. Quach H, et al. Genetic adaptation and neandertal admixture shaped the immune system of human populations. Cell.2016;167(3):643-656.e17.

28. Hudson TJ. Wanted: regulatory SNPs. Nat Genet. 2003;33(4):439-440.

29. Resnick MA, Inga A. Functional mutants of the sequence-specific transcription factor p53 and implications for master genes of diversity. Proc Natl Acad Sci U S A. 2003;100(17):9934-9939.

30. Schröder NW, Schumann RR. Single nucleotide polymorphisms of Toll-like receptors and susceptibility to infectious disease. Lancet Infect Dis. 2005;5(3):156-164.

31. Barreiro LB, et al. Evolutionary dynamics of human Toll-like receptors and their different contributions to host defense. PLoS Genet. 2009;5(7):e1000562.

32. Bochud PY, Bochud M, Telenti A, Calandra T. Innate immunogenetics: a tool for exploring new frontiers of host defence. Lancet Infect Dis. 2007;7(8):531-542.

33. Miyairi I, DeVincenzo JP. Human genetic factors and respiratory syncytial virus disease severity. Clin Microbiol Rev. 2008;21(4):686-703.

34. Gagro A, et al. Increased Toll-like receptor 4 expression in infants with respiratory syncytial virus bronchiolitis. Clin Exp Immunol. 2004;135(2):267-272.

35. Monick MM, et al. Respiratory syncytial virus up-regulates TLR4 and sensitizes airway epithelial cells to endotoxin. J Biol Chem. 2003;278(52):53035-53044.

36. Rudd BD, Burstein E, Duckett CS, Li X, Lukacs NW. Differential role for TLR3 in respiratory syncytial virus-induced chemokine expression. J Virol. 2005;79(6):3350-3357.

37. Mailaparambil B, Krueger M, Heinze J, Forster J, Heinzmann A. Polymorphisms of toll like receptors in the genetics of severe RSV associated diseases. Dis Markers. 2008;25(1):59-65.

38. Janssen R, et al. Genetic susceptibility to respiratory syncytial virus bronchiolitis is predominantly associated with innate immune genes. J Infect Dis. 2007;196(6):826-834.

39. Xie XH, Law HK, Wang LJ, Li X, Yang XQ, Liu EM. Lipopolysaccharide induces IL-6 production in respiratory syncytial virus-infected airway epithelial cells through the toll-like receptor 4 signaling pathway. Pediatr Res. 2009;65(2):156-162.

40. Scagnolari C, et al. Gene expression of nucleic acid-sensing pattern recognition receptors in children hospitalized for respiratory syncytial virus-associated acute bronchiolitis. Clin Vaccine Immunol. 2009;16(6):816-823.

41. Bendelja K, et al. Decreased Toll-like receptor 8 expression and lower TNF- $\alpha$ synthesis in infants with acute RSV infection. Respir Res. 2010;11:143.

42. Eckardt-Michel J, Lorek M, Baxmann D, Grunwald T, Keil GM, Zimmer G. The fusion protein of respiratory syncytial virus triggers p53-dependent apoptosis. J Virol. 2008;82(7):3236-3249.

43. Groskreutz DJ, et al. Respiratory syncytial virus decreases $\mathrm{p} 53$ protein to prolong survival of airway epithelial cells. J Immunol. 2007;179(5):2741-2747.

44. Machado D, et al. Role of $\mathrm{p} 53 / \mathrm{NF}-\kappa \mathrm{B}$ functional balance in respiratory syncytial virus-induced inflammation response. J Gen Virol. 2018;99(4):489-500.

45. Martínez I, et al. Induction of DNA double-strand breaks and cellular senescence by human respira- tory syncytial virus. Virulence. 2016;7(4):427-442.

46. Ehrnström B, et al. Toll-like receptor 8 is a major sensor of group B Streptococcus but not Escherichia coli in human primary monocytes and macrophages. Front Immunol. 2017;8:1243.

47. Cervantes JL, Weinerman B, Basole C, Salazar JC. TLR8: the forgotten relative revindicated. Cell Mol Immunol. 2012;9(6):434-438.

48. Wang CH, Eng HL, Lin $\mathrm{KH}$, Liu HC, Chang CH, Lin TM. Functional polymorphisms of TLR8 are associated with hepatitis $\mathrm{C}$ virus infection. Immunology. 2014;141(4):540-548.

49. Oh DY, et al. A functional toll-like receptor 8 variant is associated with HIV disease restriction. JInfect Dis. 2008;198(5):701-709.

50. Beima-Sofie KM, et al. Toll-like receptor variants are associated with infant HIV-1 acquisition and peak plasma HIV-1 RNA level. AIDS. 2013;27(15):2431-2439.

51. Davila S, et al. Genetic association and expression studies indicate a role of toll-like receptor 8 in pulmonary tuberculosis. PLoS Genet. 2008;4(10):e1000218.

52. Valverde-Villegas JM, et al. Endosomal toll-like receptor gene polymorphisms and susceptibility to $\mathrm{HIV}$ and $\mathrm{HCV}$ co-infection - differential influence in individuals with distinct ethnic background. Hum Immunol. 2017;78(2):221-226.

53. Dunford A, et al. Tumor-suppressor genes that escape from $\mathrm{X}$-inactivation contribute to cancer sex bias. Nat Genet. 2017;49(1):10-16.

54. Libert C, Dejager L, Pinheiro I. The X chromosome in immune functions: when a chromosome makes the difference. Nat Rev Immunol. 2010;10(8):594-604

55. Deng X, Berletch JB, Nguyen DK, Disteche CM. $\mathrm{X}$ chromosome regulation: diverse patterns in development, tissues and disease. Nat Rev Genet. 2014;15(6):367-378.

56. Jurk M, et al. Human TLR7 or TLR8 independently confer responsiveness to the antiviral compound R-848. Nat Immunol. 2002;3(6):499.

57. Shatz M, Menendez D, Resnick MA. The human TLR innate immune gene family is differentially influenced by DNA stress and p53 status in cancer cells. Cancer Res. 2012;72(16):3948-3957.

58. Lu H, et al. VTX-2337 is a novel TLR8 agonist that activates NK cells and augments ADCC. Clin Cancer Res. 2012;18(2):499-509.

59. Dietsch GN, et al. Late-stage cancer patients remain highly responsive to immune activation by the selective TLR8 agonist motolimod (VTX2337). Clin Cancer Res. 2015;21(24):5445-5452.

60. Peng G, et al. Toll-like receptor 8-mediated reversal of CD4+ regulatory T cell function. Science. 2005;309(5739):1380-1384.

61. Shatz M, Shats I, Menendez D, Resnick MA. p53 amplifies Toll-like receptor 5 response in human primary and cancer cells through interaction with multiple signal transduction pathways. Oncotarget. 2015;6(19):16963-16980.

62. Luo H, et al. Zika, dengue and yellow fever viruses induce differential anti-viral immune responses in human monocytic and first trimester trophoblast cells. Antiviral Res. 2018;151:55-62.

63. Zitvogel L, Galluzzi L, Kepp O, Smyth MJ, Kroemer G. Type I interferons in anticancer immunity. Nat Rev Immunol. 2015;15(7):405-414. 